\title{
Versicherungsmedizinische Kompetenz per Mausklick für den ärztlichen Alltag
}

\author{
Verena Renggli, \\ Yvonne Bollag, \\ Christian Schmidts, \\ Martina Lehr, \\ Caroline Brugger, \\ Regina Kunz
}

Im Auftrag der Schweizerischen Gesellschaft der Vertrauens- und Versicherungsärzte, SGV, und der Swiss Insurance Medicine, SIM

\section{Korrespondenz:}

Verena Renggli Referenzstelle Bildung asim, Versicherungsmedizin Universitätsspital Basel Petersgraben 4

CH-4031 Basel

Tel. 0612655568

Fax 0612655534
Sie sind Assistenzarzt und stets im Zeitdruck. Eine Ihrer Patientinnen hat eine seltene Erkrankung und benötigt zur Behandlung ein Medikament, das die Krankenkasse nicht ohne vorgängige Kostengutsprache bezahlt. Sie müssen einen Antrag für eine Kostenübernahme an den Vertrauensarzt der Krankenkasse schreiben. Wissen Sie, wie Sie vorgehen müssen? Oder fühlen Sie sich etwas überfordert? Es gibt eine Lösung per Mausklick: das eLearning Curriculum Versicherungsmedizin

\section{Wo treffen Ärzte im Praxisalltag auf versicherungsmedizinische Themen?}

Im medizinischen Alltag sind praktizierende Ärzte regelmässig mit Fragen konfrontiert, die an der Schnittstelle «Gesundheitsproblem ihrer Patienten / rechtlicher Rahmen von Sozial- und Privatversicherungen» entstehen. Als behandelnder Arzt kennen Sie solche Situationen:

- Auf Ihrer heutigen Sprechstundenliste steht eine junge Patientin mit einer längerdauernden psychischen Störung, die mit Ihnen die Möglichkeit einer IV-Anmeldung besprechen möchte.

- Ein Patient bittet Sie, ihm wegen chronischer Rückenschmerzen ein Arbeitsunfähigkeitszeugnis zu schreiben. Am nächsten Tag beschwert sich der Arbeitgeber und unterstellt Ihnen ein Gefälligkeitszeugnis.

- Auf Ihrem Schreibtisch liegt ein Stapel von Vordrucken, mit denen Versicherungen detaillierte Informationen über Ihre Patienten erfassen wollen. Dürfen Sie als behandelnder Arzt diese Infos überhaupt herausgeben?

In all diesen Fragen arbeiten Sie mit Versicherungen zusammen. Fehlt Ihnen das fundierte Grundwissen, um für Ihre Patienten an der Schnittstelle zu den Versicherungen kompetente und professionelle Auskünfte zu erteilen oder Handlungen vorzunehmen, können Sie sich überfordert fühlen. Gerade junge Assistenzärzte finden sich schnell in solchen Situationen und werden inaktiv oder gehen fehlerhaft vor. Für sie wurde im Auftrag der Swiss Insurance Medicine, SIM, und der Schweizerischen Gesellschaft der Vertrauens- und Versicherungsärzte, SGV, das eLearning Curriculum Versicherungsmedizin erstellt.

\section{Was ist Versicherungsmedizin?}

Versicherungsmedizin umfasst die Anwendung von handlungsbezogenem medizinischem Wissen und

\section{Compétences en matière de médecine des assurances à portée de souris}

Sur mandat de la Société suisse des médecins-conseils et médecins d'assurances (SSMC) et de Swiss Insurance Medicine (SIM), la Swiss Academy of Insurance Medicine (asim) a développé un cursus interactif destiné aux médecins en formation et aux médecins installés. L'objectif de cette formation est de permettre aux médecins d'acquérir des connaissances de base en médecine des assurances afin de maîtriser les questions liées aux cas d'assurance en toute confiance et avec compétence au quotidien.

Conçue sous forme modulaire, cette formation englobe l'assurance-maladie, l'assurance-accidents et invalidité ainsi que les assurances privées et porte notamment sur le certificat médical, l'expertise, le case management et la profession de médecin d'assurances. L'enseignement est dispensé à l'aide de nombreux exemples pratiques tirés du quotidien et organisé de manière interactive. La méthode d'apprentissage en ligne permet aux médecins d'intégrer la formation dans leur quotidien et de mettre également à profit de petites plages de temps libre à cet effet. Grâce à une connexion directe à Internet, les médecins ont accès à divers formulaires, tableaux et notices qui leur seront utiles tous les jours. La formation peut actuellement être suivie gratuitement à l'adresse www.henet.ch/ asim-basel (en allemand). Il est prévu de compléter la formation en ligne par des cours facultatifs en présence portant sur des aptitudes précises, par ex. l'évaluation de la capacité de travail, et permettant une discussion et un échange entre pairs sur des thèmes particuliers.

Kompetenzen, medizinischen Methoden und ärztlichen Werten, um für (potentiell) betroffene Menschen kollektive Regelungen zu entwickeln und umzusetzen, die negative gesundheitliche und soziale Konsequenzen von Krankheit und Unfall abfedern helfen. Sie beinhaltet u. a. folgende Themenschwerpunkte:

- Krankschreibung und Förderung der Rückkehr zur Arbeit; 
- Begutachtung von Langzeit-Arbeitsunfähigkeit;

- Steuerung im Gesundheitssystem und der Krankenversorgung;

- Risikoeinschätzung für Lebensversicherung;

- Förderung von Teilhabe in der Gesellschaft jenseits des Arbeitslebens.

\section{Die Ziele des Curriculums}

Das eLearning Curriculum Versicherungsmedizin zielt darauf ab, praxisrelevantes Grundwissen der Versicherungsmedizin zu vermitteln, damit die behandelnden Ärzte ihre Patienten im Praxisalltag an der Schnittstelle zu den Versicherungen sicher und kompetent begleiten können. Dabei bilden Fallbeispiele aus der täglichen Praxis die Grundlage der einzelnen Module. Das Curriculum richtet sich an Assistenzärzte im zweiten und dritten Weiterbildungsjahr, und auch an alle praktizierenden Ärzte, die sich solides Grundwissen der Versicherungsmedizin aneignen wollen.

\section{Entwicklung und Aufbau}

Eine Arbeitsgruppe mit Vertretern der Schweizerischen Gesellschaft der Vertrauens- und Versicherungsärzte, SGV, der Swiss Insurance Medicine, SIM , und der Swiss Academy of Insurance Medicine, asim, sowie einem Vertreter der Lehrbeauftragten der schweizerischen Universitäten für das Fach Versicherungsmedizin hat gemeinsam einen Katalog für den postgradualen Erwerb allgemeiner versicherungsmedizinischer Kompetenzen im Rahmen der Weiterbildung zum klinischen Facharzt entwickelt. In Fokusgruppen mit niedergelassenen Ärzten und Interviews mit Experten der grossen Versicherungszweige haben die Autoren des Curriculums erhoben, wo Defizite wahrgenommen werden und ein Bedürfnis nach mehr Wissen und besseren Fertigkeiten besteht. In mehreren iterativen Schleifen haben Versicherungsmediziner und Juristen die Lerninhalte

\section{Abbildung 1}

Bildschirmansicht aus dem Modul Krankenversicherung.

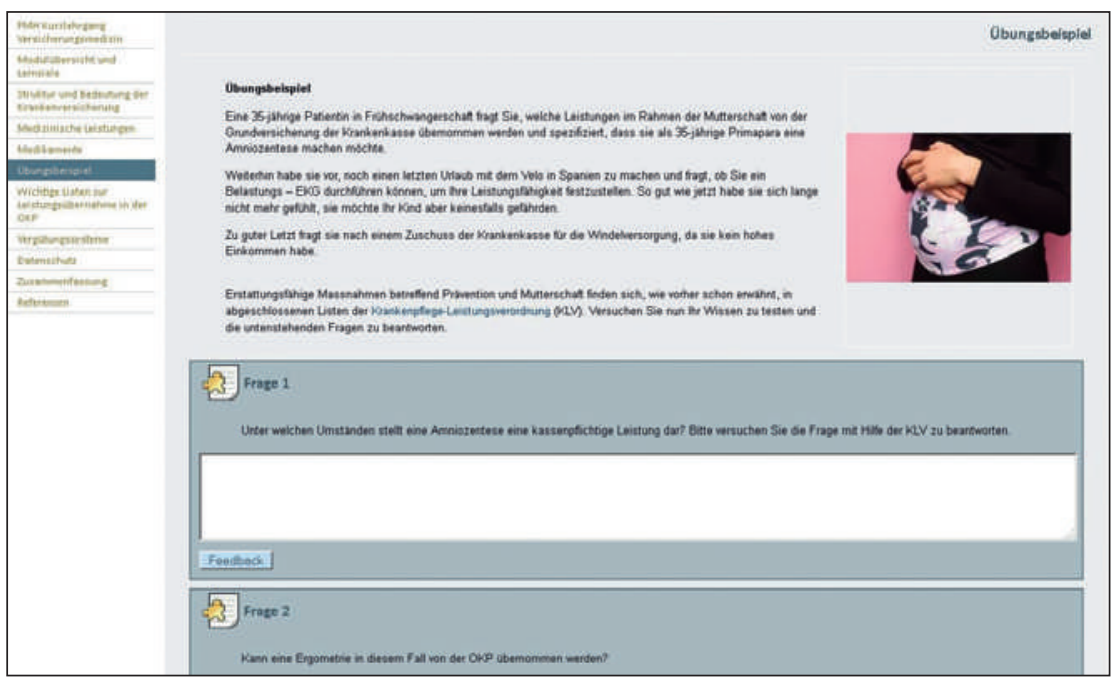

ausgearbeitet. Fachexperten verschiedener Institute haben die Inhalte reviewt, praktizierende Ärztinnen und Ärzte die Module pilotiert und kommentiert. Das Feedback wurde daraufhin wieder in das Curriculum eingepflegt.

Das eLearning Curriculum gliedert sich in neun Module. Jedes Modul ist in sich abgeschlossen, enthält aber Querverweise zu den anderen Modulen.

Einführung in die Versicherungsmedizin: Mit Grundkenntnissen über die soziale Sicherheit und die Sozialversicherungssysteme der Schweiz verbessern Sie Ihr Verständnis für das Umfeld der Versicherungsmedizin.

Die Krankenversicherung: Bezahlt die obligatorische Krankenpflegeversicherung OKP die Behandlung von Haltungsschäden? Welche Medikamente kann die Grundversicherung der Krankenkasse übernehmen? Welche Informationen dürfen Sie an die Krankenkasse weitergeben? Diese Informationen und viele andere finden Sie in diesem Modul.

Das Arztzeugnis: Ihre Patientin hat ein chronisches Rückenleiden und kann ihrer Arbeit als Verkäuferin nur noch sehr eingeschränkt nachgehen. Bei der Ausstellung eines detaillierten Arztzeugnisses müssen Sie als behandelnder Arzt jedoch nicht nur die Einschränkungen, sondern auch die verbleibenden Fähigkeiten und Ressourcen Ihrer Patientin beurteilen. Hier lernen Sie das notwendige versicherungsmedizinische Know-how.

Die Unfallversicherung: Erleidet Ihr Patient einen Unfall, so hat er Anspruch auf andere Versicherungsleistungen als bei einer Krankheit. Dies kann für ihn von zentraler Bedeutung sein. Kennen Sie die Unterschiede? In diesem Modul finden Sie die Antworten.

Die Invalidenversicherung: Die Invalidenversicherung hat neue Instrumente für die Früherfassung/ Frühintegration von Menschen mit Beeinträchtigungen eingeführt. Die IV-Stelle Ihres Kantons empfiehlt Ihnen, Patienten mit gehäufter oder längerer Arbeitsunfähigkeit frühzeitig zur Meldung aufzufordern, um eine frühe Reintegration in den Arbeitsprozess zu unterstützen. Wie gehen Sie vor? In diesem Modul werden die entscheidenden Schritte des IV-Verfahrens beschrieben und die wichtigsten Formulare, inklusive ausgefüllter Beispiele, vorgestellt.

Die Privatversicherung: Ihre Patientin möchte eine Lebensversicherung abschliessen und bittet Sie um eine ärztliche Untersuchung, eine Vorbedingung für den Versicherungsabschluss. Welche Daten müssen, welche dürfen Sie bekanntgeben? Informiert wird über die wichtigsten Schnittstellen zwischen Ärzten und Privatversicherungen und über den Datenschutz.

Case Management: Vor der Türe wartet der Case Manager der SUVA und möchte mit Ihnen als behandelnder Ärztin die berufliche Wiedereingliederung ihrer verunfallten Patientin koordinieren. Das Modul erklärt, wie Case Management funktioniert, und zeigt auf, wo und wie Sie beim Case Management entscheidend zur Wiedereingliederung beitragen können. 
Abbildung 2

Bildschirmansicht der Übungsfragen zum Modul Invalidenversicherung.

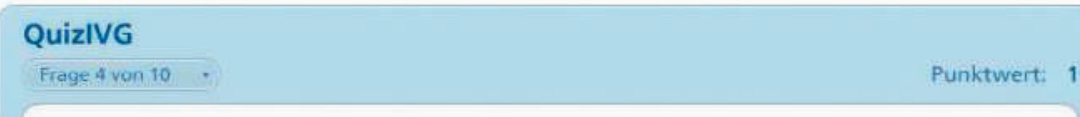

Sie als behandelnder Arzt können Ihren Patienten, nach Rücksprache mit ihm, bei der IV anmelden. Ist diese Aussage richtig oder falsch?

Richtig

Falsch Das ist nicht korrekt.

Wahrend eine Meldung bei der IV nicht zwingend von der hetroffenen Person selhst gemacht werden muss, kann eine IV-Anmeldung nur durch den Patienten selbst erfolgen.

Nä́chste Frage
Begutachtung: Die kantonale IV-Stelle fordert Ihre Patientin auf, sich einer Begutachtung bei einer unabhängigen polydisziplinären Gutachterstelle zu unterziehen. Ihre Patientin ist verunsichert und befragt Sie in der nächsten Konsultation über das Vorgehen und ihre Rechte und Pflichten. Was können Sie antworten? Hilfe findet sich in diesem Modul.

Berufsfeld Versicherungsmedizin: Im Bereich Versicherungsmedizin gibt es für Sie als Arzt verschiedene professionelle medizinische Rollen, die hier vorgestellt werden, zudem informiert das Modul über Bildungsangebote und wichtige Organisationen, Institutionen und Fachverbände.

\section{Wie funktioniert das eLearning Programm?}

Das Curriculum ist auf einer webbasierten Plattform aufgeschaltet und zurzeit frei zugänglich (www. henet.ch/asim-basel). Die Startseite listet die einzelnen Module auf, die Sie einzeln anwählen können. Jedes Modul wiederum ist in Kapitel gegliedert. Die Kapitelübersicht links (Abb. 1) ist direkt mit dem entsprechenden Inhalt verlinkt. Dies ermöglicht Ihnen, auch zu einem späteren Zeitpunkt gezielt Informationen wiederzufinden und das eLearning Curriculum als Nachschlagewerk zu nutzen.

Bei der Ausgestaltung wurde grosser Wert auf den Praxisbezug gelegt. Entsprechend nimmt die Theorie immer wieder Bezug auf konkrete Situationen aus der Patientenversorgung. Regelmässige Reflexionsfragen involvieren die Lernenden aktiv und lassen sie das soeben Gelernte anwenden. Zur Überprüfung des eigenen Wissenszuwachses wurden für die meisten Module auch einige Übungsfragen zusammengestellt (Abb. 2).
Zum Durcharbeiten eines Moduls benötigen Sie, abhängig vom Thema und der Intensität Ihres Lernens, zwischen 30 bis 90 Minuten. Dabei können Sie die Module jederzeit unterbrechen und zu einem späteren Zeitpunkt fortsetzen.

\section{eLearning hat Vorteile}

Die Hauptmotivation für die Wahl der eLearning Methode lag in der hohen Flexibilität des Mediums: Es erlaubt den praktizierenden Ärzten, die Lernaktivitäten in den Berufsalltag oder in die knappe Weiterbildungszeit zu integrieren und auch kleine, freie Zeitfenster zu nutzen, ohne dass Absenzen vom Arbeitsplatz entstehen. Themenschwerpunkte und Lerngeschwindigkeit orientieren sich am Vorwissen und dem individuellen Wissensbedarf.

Das eLearning erlaubt den Lernenden, die diversen Formulare, Merkblätter und Tabellen durch entsprechende Verlinkung direkt im Internet abzurufen und im Praxisalltag einzusetzen. Ebenfalls verlinkt sind die relevanten Gesetzesartikel, die bei Interesse nachgelesen werden können, sowie weiterführende Literatur. Durch einen direkten Zugang zu einem Glossar der Versicherungsmedizin kann der Lernende unbekannte Begriffe nachlesen und Halbwissen klären.

\section{eLearning wirkt}

Wie effektiv ist eLearning als Lernmethode? Eine neuere Metaanalyse zeigt für Internet-basiertes Lernen bei Gesundheitsberufen einen grossen Nutzen [1]. Im Vergleich zu anderen Formen des «nicht-Internetbasierten Lernens», z.B. in Form von Präsenzveranstaltungen, Gruppenunterricht, oder mit schriftlichem Lehrmaterial, konnte ein ebenbürtiger bis überlegener Lernerfolg [1-3] erzeugt werden. Andere Studienergebnisse weisen darauf hin, dass eLearning sogar ein effizienteres Lernen erzielt [2, 3].

\section{Ausblick}

Das eLearning Curriculum Versicherungsmedizin ist abgeschlossen und kommt jetzt in die Praxisphase. Geplant ist, das eLearning mit einem Präsenzteil zu ergänzen, in dem Fertigkeiten, wie z.B. die Beurteilung der Arbeitsunfähigkeit, vermittelt werden. Lernen in Kleingruppen an spezifischen Fällen und im Austausch mit Kollegen soll auch hier eine hohe Praxisrelevanz sicherstellen.

\section{Literatur}

1 Cook DA, Levinson AJ, Garside S, Dupras DM, Erwin PJ, Montori VM. Internet-based learning in the health professions - a meta-analysis. JAMA. 2008;300:1181-96.

2 Ruf D, Berner MM, Kriston L, Härter M. E-Learning eine wichtige Unterstützung in der medizinischen Aus-, Fort- und Weiterbildung? Bundesgesundheitsbl Gesundheitsforsch - Gesundheitsschutz. 2008;51:1061-9.

3 Henning PA, Schnur A. eLearning in continuing medical education: A comparison of knowledge gain and learning efficiency. J Of Med Marketing. 2009;9:156-60. 